ARtigo de ReVISÃo

Review ArTicle

\title{
Tratamento da Arterite de Takayasu
}

\section{Takayasu's Arteritis Treatment}

\author{
Alexandre Wagner Silva de Souza ${ }^{(1)}$, Raissa Maria Sampaio Neves ${ }^{(1)}$, Kathia Regina Oliveira ${ }^{(1)}$, \\ Emília Inoue Sato ${ }^{(2)}$
}

\section{RESUMO}

A arterite de Takayasu (AT) é uma vasculite granulomatosa crônica que envolve a aorta e seus principais ramos. A monitoração da atividade da doença e o melhor esquema terapêutico ainda têm sido um desafio para todos os que tratam estes pacientes. Corticosteróides e imunossupressores vêm sendo utilizados na prática clínica diária com resultados nem sempre animadores. Apesar de não haver estudos controlados que abordem o tratamento da AT, diferentes estudos observacionais descrevem a resposta de pacientes com AT ao uso de corticosteróides, metotrexato (MTX), azatioprina e ciclofosfamida. Após a introdução da terapia biológica, novas perspectivas têm surgido para os pacientes com AT refratários aos esquemas terapêuticos tradicionais.

Palavras-chave: arterite de Takayasu, tratamento, metotrexato, azatioprina, ciclofosfamida, terapia biológica.

\section{INTRODUÇÃO}

A arterite de Takayasu (AT) é uma doença inflamatória crônica, de causa desconhecida, que envolve artérias de grande e de médio calibres, incluindo a aorta e seus principais ramos, além de artérias pulmonares e coronárias ${ }^{(1)}$. $\mathrm{Na}$ AT ocorre inflamação granulomatosa transmural que pode causar estenose, oclusão, dilatação e/ou formação de aneurismas nas artérias envolvidas ${ }^{(2)}$. A AT é uma doença rara que acomete principalmente mulheres em fase reprodutiva. Tem maior incidência em países asiáticos, mas já foi descrita em todos os grupos étnicos e em diferentes regiões geográficas do planeta ${ }^{(3)}$.

De forma didática, o quadro clínico da AT inclui uma fase inflamatória sistêmica, associada à febre e aos sintomas constitucionais, uma fase de inflamação vascular, e uma última fase tardia de isquemia. Entretanto, nem sempre todas as fases são observadas seqüencialmente em todos

\begin{abstract}
Takayasus's arteritis is a chronic granulomatous vasculitis involving the aorta and its main branches. Monitoring disease activity and the choice for the best therapy has been major challenger faced by all physicians who treat these patients. Corticosteroids and immunosuppressive therapy have been used in daily medical practice, but results have not always been encouraging. Although there are no controlled studies evaluating the treatment of Takayasu's arteritis, observational studies have described the response to the use of corticosteroids, methotrexate, azathioprine and cyclophosphamide. The emerging of biological therapy has brought new perspective for Takayasu's patients who are refractory to conventional therapy.
\end{abstract}

Keywords: Takayasu's arteritis, treatment, methotrexate, azathioprine, cyclophosphamide, biological therapy.

os pacientes $^{(4)}$. Não há um marcador adequado para se mensurar a atividade da AT. A velocidade de hemossedimentação (VHS) e a quantificação da proteína $\mathrm{C}$ reativa (PCR) são utilizadas na prática clínica diária, mas nem sempre apresentam boa correlação com a atividade da doença. Os critérios de atividade mais utilizados para guiar a terapêutica são os publicados pelo National Institutes of Health (NIH) (Tabela 1$)^{(4)}$. Entretanto, a experiência do próprio NIH demonstra que esses critérios apresentam falhas na detecção de atividade da doença. Entre pacientes considerados em remissão, submetidos a procedimentos cirúrgicos vasculares, foram observados sinais histopatológicos de inflamação na artéria operada em $44 \%$ dos casos. Avaliações arteriográficas seqüenciais em pacientes com AT fora de atividade demonstraram que $61 \%$ apresentaram novas lesões arteriais, num período de seguimento médio de 17,5 meses $^{(4-5)}$.

Disciplina de Reumatologia da Escola Paulista de Medicina da Universidade Federal de São Paulo (EPM-UNIFESP). Recebido em 05/01/05. Aprovado, após revisão, em 17/08/05.

1. Pós-graduando da Disciplina de Reumatologia da EPM-UNIFESP.

2. Professora Titular da Disciplina de Reumatologia da EPM-UNIFESP.

Endereço para Correspondência: Alexandre Wagner Silva de Souza, Rua Botucatu, 740, $3^{\circ}$ andar, São Paulo, CEP 04023-062, SP, Brasil,

e-mail: alexandre_wagner@uol.com.br 
TABELA 1

Critérios de atividade para a AT, SEgundo o NIH ${ }^{(4)}$

Queixas sistêmicas

Elevação da VHS

Piora da isquemia (claudicação, diminuição ou ausência de pulso, sopro, dor sobre grandes vasos ou assimetria de pressão arterial)

Novas alterações arteriográficas

Para se considerar AT em atividade, o paciente deve apresentar 0 aparecimento ou a exacerbação de dois ou mais parâmetros acima.

AT - arterite de Takayasu

NIH - National Institutes of Health

VHS - velocidade de hemossedimentação

Recentemente, a angiorressonância vem sendo estudada na AT como método de avaliação na atividade da doença. Apresenta vantagens por ser um exame que não requer punção arterial, não utiliza contraste iodado e o paciente não é exposto à radiação ionizante. Além disso, a angiorressonância pode fornecer informações a respeito da anatomia da parede do vaso com boa correlação com a angiografia. Seqüências ponderadas em T2 e o STIR captam a presença de edema na parede da artéria, o que poderia significar sinal de inflamação ativa ${ }^{(6-8)}$. Em estudo que avaliou a correlação de sinais de edema na parede arterial com sinais clínicos de atividade na AT, foi observada alta freqüência de edema na parede do vaso (94\%) em pacientes considerados em atividade. Entretanto, entre pacientes ditos fora de atividade e naqueles com atividade da doença incerta, mais de $50 \%$ apresentam edema na parede do vaso. Tso et a ${ }^{(9)}$ sugerem que o achado de edema na parede do vaso pode não significar apenas inflamação e pode persistir após a resolução do processo inflamatório, na fase de reparação. Tais achados demonstram que a angioressonância isoladamente ainda não pode ser considerada um marcador de atividade na AT. Outro fator que devemos levar em consideração, sobretudo em nosso meio, é o alto custo da angioressonância.

O tratamento da AT inclui o uso de corticosteróides em altas doses (1-2 mg/Kg/dia) na fase ativa da doença por um a três meses. Imunossupressores são utilizados em caso de reativação da doença, após o desmame dos corticosteróides e quando não há resposta adequada a estes. Inicialmente, a ciclofosfamida por via oral em associação aos corticosteróides foi uma das alternativas utilizadas pelos investigadores do NIH. A partir de 1987, novas terapias para as vasculites sistêmicas passaram a ser utilizadas, pois a ciclofosfamida por tempo prolongado apresenta alta freqüência de toxicidade. O metotrexato (MTX) e a azatioprina passaram a ser utilizados como alternativa de imunossupressão na AT. O tratamento da AT tem sido baseado em experiência clínica retrospectiva, e, em série de casos, pois não há ensaios clínicos controlados e randômicos avaliando a terapêutica nesta rara entidade clínica ${ }^{(5)}$. Recentemente, novas alternativas vêm sendo utilizadas nos casos refratários de $\mathrm{AT}$, em pequenas séries de casos ou em relatos anedóticos. Micofenolato mofetil, leflunomida, infliximabe e etanercepte encontramse entre as novas promessas no tratamento AT.

Nesse artigo os autores se propõem a revisar e relatar séries de casos e estudos retrospectivos que avaliaram diversos regimes terapêuticos na AT. Foi realizada a pesquisa no banco de dados do LILACS e do MEDLINE para a busca de artigos que abordassem esse tema.

\section{CORTICOSTERÓIDES E METOTREXATO}

A despeito da terapia com corticosteróides, um significante número de pacientes com AT apresenta doença crônica ou recidivante ${ }^{(5,10)}$. Em estudo prospectivo realizado pelo NIH, com observações por um período superior a 31 anos, 60 pacientes foram incluídos. Vinte e nove de $48(60 \%)$ tratados com corticosteróides e 10 de $25(40 \%)$ tratados com associação corticosteróide e imunossupressor (ciclofosfamida ou azatioprina) obtiveram remissão pelo menos uma vez. Contudo, $45 \%$ de todos os pacientes tinham no mínimo uma recidiva e $23 \%$ nunca entraram em remissão nem puderam suspender o uso de corticosteróide ${ }^{(10)}$.

Em 1989, foi publicado o primeiro relato de caso com sucesso ao uso de MTX em baixas doses em mulher de 20 anos que já havia sido submetida a seis angioplastias para alívio dos sintomas isquêmicos. Após três semanas do uso de MTX, sem corticosteróide, numa dose de 7,5 mg/semana, houve melhora da claudicação no braço esquerdo, devido à estenose na artéria subclávia. Nas duas vezes em que a paciente suspendeu a medicação, os sintomas retornaram ${ }^{(11)}$. Apenas um estudo prospectivo avaliou a utilidade do MTX na AT. Neste estudo foram incluídos apenas pacientes com comprovação angiográfica de comprometimento de artérias de grande calibre. Inicialmente participaram 18 pacientes considerados relativamente resistentes ao uso de corticosteróides. Durante o estudo ocorreram duas perdas de seguimento e apenas 16 pacientes foram seguidos por um período médio de 2,8 anos. O MTX foi iniciado na dose de $0,3 \mathrm{mg} / \mathrm{kg} / \mathrm{semana}$ ou no máximo $15 \mathrm{mg} / \mathrm{semana}$. Esta dose foi gradualmente aumentada em $2,5 \mathrm{mg} / \mathrm{semana}$ a cada 1 a 2 semanas, até a dose máxima de $25 \mathrm{mg} /$ semana. A dose inicial de prednisona foi de $1 \mathrm{mg} / \mathrm{kg}$, com redução após um mês. Seis destes pacientes já haviam utilizado, antes do estudo, ciclofosfamida ou azatioprina como terapia alternativa sem sucesso. A associação de corticosteróide 
com MTX levou à remissão em $81 \%$ dos casos $(13$ dos 16 pacientes). Porém, sete pacientes apresentaram recidiva após a retirada do corticosteróide e foram novamente tratados com o mesmo esquema. O corticosteróide foi interrompido com sucesso após um período de 2, 25 e 28 meses, respectivamente, em apenas três destes sete pacientes. Oito pacientes $(50 \%)$ obtiveram remissão por um período médio de 18 meses e conseguiram ficar sem utilizar corticosteróide por aproximadamente 14,4 meses. Somente um quarto dos pacientes sustentou a remissão por um ano, sem medicação. Os autores concluíram que mais estudos são necessários para determinar quanto tempo de uso de MTX seria necessário para induzir remissão, se o curso da doença é alterado por esse tratamento ou se diferentes esquemas terapêuticos são superiores ao utilizado nesse estudo aberto ${ }^{(10)}$.

\section{AZATIOPRINA E CICLOFOSFAMIDA}

A maior experiência com o uso destas drogas também advém de estudos do NIH. Sessenta pacientes foram seguidos por um período de 6 meses a 20 anos (média de 5,3 anos). $\mathrm{O}$ tratamento foi realizado inicialmente com prednisona (1 mg/kg/dia) por 1-3 meses, com posterior redução da dose. A remissão foi alcançada em $60 \%$ dos pacientes, porém metade deles apresentou recorrência da doença durante o seguimento. Os imunossupressores foram utilizados nos pacientes que permaneceram em atividade, apesar dos corticosteróides, ou que tiveram recorrência da doença, após a remissão. Nesses casos, os imunossupressores utilizados foram o MTX $(0,15-0,3 \mathrm{mg} / \mathrm{kg} / \mathrm{semana})$ e a ciclofosfamida ( $2 \mathrm{mg} / \mathrm{kg} /$ dia), de acordo com o julgamento clínico. Entre os pacientes que utilizaram drogas imunossupressoras, $40 \%$ alcançaram remissão da doençç ${ }^{(5)}$.

A experiência japonesa é semelhante à descrita pelo $\mathrm{NIH}$. Ito et al ${ }^{(12)}$ demonstraram que dos 150 pacientes em seguimento, 29 precisaram utilizar alguma droga imunossupressora (azatioprina, ciclofosfamida ou mercaptopurina). Nos que receberam imunossupressores, uma boa resposta foi observada em apenas $29 \%$, resposta pobre foi descrita em $33 \%$ e a droga foi ineficaz em $29 \%$ dos casos.

A azatioprina é uma droga que vem sendo utilizada como alternativa ao MTX e à ciclofosfamida. Há poucos estudos na literatura e o mais interessante deles é um estudo aberto feito por Valsakumar et $a^{(13)}$, na Índia. Os autores selecionaram 15 pacientes recém diagnosticados e com a doença em atividade, segundo critérios estabelecidos pelos mesmos. Os pacientes iniciavam o tratamento com azatioprina $(2 \mathrm{mg} / \mathrm{kg} / \mathrm{dia})$ associado à prednisolona $(1 \mathrm{mg} / \mathrm{kg} / \mathrm{dia})$ e o tratamento era mantido por um ano. A dose do corticosteróide era reduzida de forma gradativa, a partir da sexta semana, até uma dose de manutenção de $5-10 \mathrm{mg} /$ dia. Essa redução poderia ser realizada de forma mais lenta, se houvesse atividade da doença. Foram avaliados os sintomas sugestivos de inflamação ou isquemia vascular, provas de atividade inflamatória incluindo VHS e PCR e angiografia. Em três meses de tratamento, todos os pacientes tiveram resolução dos sintomas sistêmicos e as provas de atividade inflamatória haviam normalizado em 13/15 (76,7\%) dos pacientes. Esses sinais de melhora se mantiveram em um ano de tratamento. Com relação à angiografia, não houve mudança significativa em nenhum paciente. Os autores concluíram que esse regime terapêutico foi bem tolerado e efetivo em melhorar os sintomas sistêmicos e os parâmetros laboratoriais de atividade ${ }^{(13)}$.

Estudos controlados e com tempo de seguimento maior são necessários para estabelecer o papel da azatioprina e da ciclofosfamida no tratamento da AT, bem como para definir qual a melhor opção, tempo e dose de tratamento. No momento, estas drogas permanecem como opções viáveis, sendo utilizadas principalmente como opção naqueles pacientes que não respondem ou que têm contra-indicações ao uso do MTX, porém com potencial terapêutico limitado ${ }^{(14)}$.

\section{NOVAS PERSPECTIVAS}

Novas alternativas à terapia convencional da AT vêm sendo utilizadas experimentalmente e de forma não controlada em alguns serviços. Dentre elas destacam-se o uso de micofenolato mofetil, leflunomide, infliximabe e etanercepte. Os pacientes estudados nas séries de casos que avaliaram o uso desses novos medicamentos apresentavam em geral uma doença refratária, a despeito do tratamento com corticosteróides e diferentes imunossupressores ${ }^{(15-18)}$.

Diana et $\mathrm{al}^{(15)}$ avaliaram três mulheres com AT, utilizando o micofenolato mofetil $2 \mathrm{~g} /$ dia, dividido em duas doses diárias. As pacientes haviam apresentado efeitos colaterais significativos com a corticoterapia prolongada e em duas delas não houve resposta ao uso de diversos imunossupressores que incluíam ciclofosfamida, ciclosporina, azatioprina e MTX, além de plasmaferese em uma paciente e de associação de ciclosporina e MTX na outra paciente. Uma das pacientes só havia utilizado corticosteróides. Após o início do micofenolato mofetil houve resolução dos sintomas constitucionais em três a quatro semanas nas três pacientes. Todas conseguiram fazer o desmame da prednisona. As três pacientes mantiveram boa resposta com 
o micofenolato mofetil em acompanhamento de 11 a 15 meses. Este foi o único relato de casos publicado avaliando o uso do micofenolato mofetil na $\mathrm{AT}^{(15)}$.

Há apenas um relato de caso publicado que avaliou o uso de leflunomida na AT. Nesta publicação, uma paciente com AT refratária ao uso de corticosteróides e MTX (20 $\mathrm{mg}$ /semana), após o início do leflunomida $30 \mathrm{mg} /$ dia, normalizou a VHS, a PCR e a proteína sérica amilóide A. Além de redução significativa dos níveis de interleucina-6 e de níveis solúveis de CD44 em sua isoforma $5^{(16)}$.

$\mathrm{O}$ infliximabe foi avaliado em duas séries de casos. Gause et a ${ }^{(17)}$ utilizaram etanercepte ou infliximabe em 7 pacientes com vasculite reumatóide, 39 com granulomatose de Wegener, $3 \mathrm{com}$ poliangiite microscópica, 5 pacientes com arterite de células gigantes e 5 pacientes com AT. Nessa série de casos, os resultados foram satisfatórios na maioria dos casos de vasculite, mas os autores não detalham a resposta clínica dos pacientes com AT.

Hoffman et $a^{(18)}$ avaliaram o infliximabe e o etanercepte em estudo aberto que incluiu 15 pacientes com AT. Nesse estudo, foram incluídos pacientes que responderam a doses altas de corticosteróides e que apresentaram recidiva da doença após redução da droga, pacientes que nunca atingiram remissão ou que apresentaram toxidade inaceitável com o uso de corticosteróides. Em 13 pacientes, um ou mais dos seguintes agentes foram utilizados antes do estudo: MTX ( 13 casos), ciclofosfamida ( 6 casos), micofenolato mofetil ( 3 casos), azatioprina ( 3 casos), ciclosporina A ( 2 casos) e tacrolimus (2 casos). Nenhum desses imunossupressores havia conseguido induzir remissão ou permitira a redução ou suspensão dos corticosteróides. Remissão sustentada foi obtida em $67 \%$ dos pacientes, ou seja, ausência de novas lesões vasculares e suspensão de corticosteróide. Essa remissão durou de 1 a 3,3 anos. Remissão parcial foi obtida em $27 \%$ dos casos, o que significa conseguir reduzir a dose do corticosteróide em $50 \%$. Apenas um paciente não respondeu ao tratamento com anti-TNF- $\alpha$ e apresentou novas lesões arteriais e não conseguiu reduzir a dose de corticosteróide $^{(18)}$.

\section{OUTROS ASPECTOS DO TRATAMENTO DA AT}

A gestação em pacientes com AT foi analisada em estudo observacional indiano. Neste estudo, foram avaliadas 24 gestações em 12 pacientes. A maioria das pacientes (91\%) apresentava hipertensão arterial sistêmica ao diagnóstico de AT. As complicações maternas tiveram relação direta com a hipertensão arterial e incluíam pré-eclampsia (quatro pacientes) e progressão para insuficiência cardíaca e para insuficiência renal (duas pacientes cada). Ocorreram dois abortos e cinco óbitos fetais intra-uterinos. O envolvimento de aorta abdominal esteve relacionado à má evolução obstétrica. Apenas o uso de alguns anti-hipertensivos e corticosteróides é permitido durante a gestação de pacientes com AT. Os imunossupressores estão contra-indicados ${ }^{(19)}$. Kerr et $\mathrm{al}^{(4)}$ observaram cinco gestações em pacientes com AT e todas evoluíram de forma satisfatória. Apenas uma paciente teve exacerbação dos sintomas durante a gestação.

Não há estudos que tenham avaliado a orientação de exercícios na AT, estes podem ser limitados pela presença de claudicação de membros, secundária a estenoses ou oclusões arteriais. A prevenção de doença aterosclerótica através da intervenção sobre fatores de risco tradicionais é importante na AT, devido à interface entre inflamação, ativação endotelial e aterosclerose. Apesar de lesões ateroscleróticas serem freqüentemente encontradas em autópsias de mulheres jovens com AT, livres de fatores de risco para doença coronariana ${ }^{(20)}$, a preocupação com estes fatores de risco deve ser mantida. O uso de antiagregantes plaquetários é bastante empregado na prática diária, apesar de não haver estudos que avaliassem eficácia na profilaxia de trombose arterial. Em estudo italiano que avaliou 104 pacientes com AT, antiagregantes foram utilizados em $84 \%$ dos pacientes, em alguma fase do acompanhamento médico. Os anticoagulantes raramente são citados nos estudos que avaliaram a terapêutica na AT e geralmente são prescritos após eventos trombóticos ou após determinadas cirurgias (por exemplo, implante de prótese valvar metálica) ${ }^{(21)}$.

$\mathrm{O}$ acometimento da artéria pulmonar na AT ocorre em aproximadamente $50 \%$ dos casos, com variação entre 14 e $86 \%^{(22-29)}$. Na maioria das vezes, esse envolvimento não é clinicamente significativo. Os sintomas, quando presentes, incluem tosse, dispnéia ou hemoptise em pequena quantidade, e podem simular tromboembolismo pulmonar ${ }^{(22)}$. Na maioria dos casos de envolvimento pulmonar na AT, o tratamento realizado foi semelhante ao das manifestações em outros sítios vasculares ${ }^{(30)}$.

A revascularização, endarterectomia e/ou a angioplastia, com ou sem a colocação de stent, são os procedimentos cirúrgicos que podem ser realizados na AT. Suas indicações incluem: hipertensão arterial na presença de estenose da artéria renal, isquemia de extremidades que limitam as atividades de vida diária, isquemia cerebral e/ou estenose crítica (>70\%) e sintomática em artérias carótidas ou vertebrais, insuficiência aórtica moderada, isquemia coronariana, 
estenose grave da aorta e dissecção ou aumento no diâmetro de aneurismas ${ }^{(31)}$.

\section{EXPERIÊNCIA BRASILEIRA}

No Ambulatório de Vasculites da Disciplina de Reumatologia da Escola Paulista de Medicina da Universidade Federal de São Paulo (EPM-UNIFESP), são utilizados os critérios de classificação do American College of Rheumatology (ACR) para $\mathrm{AT}^{(32)}$ (Tabela 2), e a definição de AT proposta pela Conferência Internacional de Chapel Hill $^{(33)}$ como métodos auxiliares no diagnóstico da AT. Os parâmetros de atividade propostos pelo $\mathrm{NIH}^{(4)}$ são rotineiramente aplicados para se aferir a atividade da doença. Falha do esquema terapêutico é considerada quando o paciente persiste com sinais clínicos de atividade da doença, apesar da otimização do tratamento, recidiva ao se reduzir a dose da medicação ou toxidade inaceitável da mesma e presença de novas lesões arteriográficas ou até mudança evolutiva do tipo arteriográfico.

TABELA 2

Critérios de ClassificaÇão da AT do ACR ${ }^{(19)}$

\begin{tabular}{|c|c|}
\hline CRITÉRIO & DEFINIÇÃO \\
\hline Idade de início $\leq 40$ anos & $\begin{array}{c}\text { Início dos sinais ou sintomas } \\
\text { sugestivos de AT em idade } \leq 40 \\
\text { anos. }\end{array}$ \\
\hline Claudicação de extremidades & $\begin{array}{l}\text { Surgimento ou exacerbação } \\
\text { de fadiga e desconforto em } \\
\text { musculatura de uma ou mais } \\
\text { extremidades aos movimentos, } \\
\text { principalmente nos membros } \\
\text { superiores. }\end{array}$ \\
\hline $\begin{array}{c}\text { Diminuição do pulso da artéria } \\
\text { braquial }\end{array}$ & $\begin{array}{l}\text { Diminuição do pulso em uma ou } \\
\text { em ambas as artérias braquiais. }\end{array}$ \\
\hline $\begin{array}{l}\text { Diferença de pressão arterial }>10 \\
\qquad \mathrm{mmHg}\end{array}$ & $\begin{array}{c}\text { Diferença de pressão arterial } \\
\text { sistólica superior a } 10 \mathrm{mmHg} \text { nos } \\
\text { membros superiores. }\end{array}$ \\
\hline $\begin{array}{c}\text { Sopro em subclávia ou aorta } \\
\text { abdominal }\end{array}$ & $\begin{array}{c}\text { Sopro observado à ausculta de } \\
\text { uma ou de ambas as artérias } \\
\text { subclávias ou na aorta abdominal. }\end{array}$ \\
\hline Alterações arteriográficas & $\begin{array}{l}\text { Estreitamento ou obstrução da } \\
\text { aorta, de seus ramos primários } \\
\text { ou de grandes artérias proximais } \\
\text { dos membros superiores ou } \\
\text { membros inferiores, excluindo- } \\
\text { se aterosclerose, displasia } \\
\text { fibromuscular e causas } \\
\text { semelhantes. As alterações são } \\
\text { geralmente focais ou segmentares. }\end{array}$ \\
\hline
\end{tabular}

AT - arterite de Takayasu

ACR - American College of Rheumatology

$\mathrm{mmHg}$ - milímetros de mercúrio
Entre 30 pacientes seguidos regularmente de 1993 a 1998, 90\% receberam prednisona, com dose média diária de 48 mg e variação de 15 a 80 mg. Em 10\% dos casos não foi necessário utilizar corticosteróides, pois a doença não apresentava sinais de atividade na época do diagnóstico ou durante o seguimento. Efeitos colaterais com o uso da prednisona foram observados em $47 \%$ dos pacientes e eles incluíam: hipertensão arterial sistêmica, fácies cushingóide, obesidade centrípeta e distúrbios do humor. Apenas 37\% dos pacientes obtiveram controle da atividade da doença somente com o uso de prednisona. MTX foi utilizado em 12 pacientes (40\%), numa dose média semanal de $8,8 \mathrm{mg}$ e variação de 5 a 15 mg. O controle da atividade da AT foi obtido em sete casos (58\%) dos pacientes que utilizaram a associação prednisona e MTX. O MTX foi descontinuado em três pacientes, devido a efeitos colaterais e foi ineficaz em dois casos. Nestes casos de falha do MTX, foi prescrito ciclofosfamida na dose de $2 \mathrm{mg} / \mathrm{Kg} /$ dia, mas também não foi obtido sucesso ${ }^{(34)}$.

Em estudo retrospectivo que avaliou dados demográficos, clínicos e arteriográficos de 73 pacientes com AT seguidos em três centros universitários brasileiros, os corticosteróides foram utilizados em $92 \%$ dos casos e imunossupressores foram necessários em 33\% dos pacientes para controlar os sinais e sintomas de atividade da AT. O imunossupressor mais utilizado foi o MTX (68\%). Além do MTX, três pacientes receberam ciclofosfamida, quatro receberam clorambucil e apenas um paciente utilizou azatioprina. Em 8\% dos casos, não foi necessário utilizar nenhuma medicação, pois a doença encontrou-se aparentemente fora de atividade durante período de seguimento ${ }^{(35)}$. Não há dados em estudos brasileiros sobre o surgimento de novas lesões arteriográficas em pacientes com AT cuja doença encontra-se aparentemente em remissão.

\section{CONCLUSÕES}

O tratamento da AT é baseado em análises observacionais e em relatos de casos. Corticosteróides e imunossupressores são amplamente utilizados na tentativa de se induzir a remissão ou de se evitar a progressão das lesões arteriais na AT, mas sem comprovação por ensaios clínicos controlados e randomizados. Grande parte da dificuldade em se produzir evidências para o tratamento da AT reside na falta de parâmetros confiáveis para se avaliar a atividade da doença e por esta entidade ser bastante rara e muitas vezes subdiagnosticada. Estudos multicêntricos com maior número de pacientes são necessários para avaliar o melhor esquema terapêutico na AT. 


\section{REFEREANCIAS}

1. Kerr GS: Takayasu's arteritis. Rheum Dis Clin North Am 21: 1014-58, 1995.

2. Seko Y: Takayasu's arteritis: insights into immunopathology. Jpn Heart J 41: 15-26, 2000.

3. Numano F: Differences in clinical presentation and outcome in different countries for Takayasu's arteritis. Curr Opin Rheumatol 9: 12-5, 1997.

4. Kerr GS, Hallahan CW, Giordano J et al: Takayasu's arteritis. Ann Intern Med 120: 919-29, 1994.

5. Hoffman GS: Takayasu's arteritis: lessons from the American National Institutes of Health experience. Int J Cardiol 54 Suppl: 83-6, 1996.

6. Cantu C, Pineda C, Barinagarrementeria F et al: Noninvasive cerebrovascular assessment of Takayasu's arteritis. Stroke 31: 2197-2202, 2000.

7. Yamada I, Nakagawa T, Himeno Y, Kobayashi Y, Numano F, Shibuya H: Takayasu's arteritis: diagnosis with breath-hold contrast-enhanced three-dimensional MR angiography. J Magn Reson Imaging 11: 481-7, 2000.

8. Kissin EY, Merkel PA: Diagnostic imaging in Takayasu's arteritis. Curr Opin Rheumatol 16: 31-7, 2004.

9. Tso E, Flamm SD, White RD, Schvartzman PR, Mascha E, Hoffman GS: Takayasu's arteritis: utility and limitations of magnetic resonance imaging in diagnosis and treatment. Arthritis Rheum 46: 1634-42, 2002.

10. Hoffman GS, Leavitt RY, Kerr GS, Rottem M, Sneller MC, Fauci AS: Treatment of glucocorticoid- resistant or relapsing Takayasu's arteritis with methotrexate. Arthritis Rheum 37: 578-582, 1994.

11. Liang GC, Nemickas R, Madayag M: Multiple percutaneous transluminal angioplasties and low dose pulse methotrexate for Takayasu's Arteritis. J Rheumatol 16: 1370-1373, 1989.

12. Ito I: Medical treatment of Takayasu's arteritis. Heart Vessels 7 Suppl: 133-7, 1992.

13. Valsakumar AK, Valappil UC, Jorapur V, Garg N, Nityanand $S$, Sinha N: Role of immunosuppressive therapy on clinical, immunological, and angiographic outcome in active Takayasu's arteritis. J Rheumatol 30: 1793-8, 2003.

14. Bahl VK, Seth S: Takayasu's arteritis revisited. Indian Heart J 54: 147-51, 2002.

15. Diana E, Schieppati A, Remuzzi G: Mycophenolate mofetil for the treatment of Takayasu's arteritis: report of three cases. Ann Intern Med 130: 422-6, 1999.

16. Haberhauer G, Feyertag J, Kittl EM, Bauer K, Dunky A: Beneficial effects of leflunomide in glucocorticoid and methotrexate-resistant Takaysus's arteritis. Clin Exp Rheumatol 19: 477-8, 2001

17. Gause A, Arbach O, Lamprecht P: Der Einsatz von TNF $\alpha$ antagonisten bei primär systemischen vaskulitiden. $Z$ Rheumatol 62: 228-34, 2003.
18. Hoffman GS, Merkel PA, Brasington RD, Lenschow DJ, Liang P: Anti-tumor necrosis factor therapy in patients with difficult to treat Takayasu's arteritis. Arthritis Rheum 50: 2296-304, 2004.

19. Sharma BK, Jain S, Vasishta K: Outcome of pregnancy in Takayasu's arteritis. Int J Cardiol 75: S159-62, 2000.

20. Numano F, Kishi Y, Tanaka A, Ohkawara M, Kakuta T, Kobayashi Y: Infammation and atherosclerosis: atherosclerotic lesions in Takayasu's arteritis. Ann N Y Acad Sci 902: 65-76, 2000.

21. Vanoli M, Daina E, Salvarani C et al. Takayasu's arteritis: a study of 104 Italian patients. Arthritis Rheum 53: 100-7, 2005.

22. Haque U, Hellmann D, Traill T, Venbrux A, Stone J: Takayasu's arteritis involving proximal pulmonary arteries and mimicking thromboembolic disease. J Rheumatol 26: 450-3, 1999.

23. Oata K: Ein seltener fall von veiderseitigem carotissubclaviaverschlus (ein beitrag zur pathologie der anastomosis peripapillaries des anges mit fehlendem radialpus). Trans Soc Path Jap 30: 680-92, 1940.

24. Nasu T: Takayasu`s truncoarteritis in Japan. Pathol Microbiol 43: 140-6, 1975.

25. Lupi E, Sanchez G, Horwitz S, Gutierrez E: Pulmonary artery involvement in Takayasu's arteritis. Chest 67: 69-74, 1975.

26. Ishikawa K: Natural history and classification of occlusive thromboaortopathy (Takayasu's disease). Circulation 57: 27-35, 1978.

27. Yamato M, Lecky J, Hiramatsu K, Kohda E: Takayasu's arteritis: radiographis and angiographic findings in 59 patients. Radiology 161: 329-34, 1986.

28. Sharma S, Kamalakar T, Rajani M, Talwar KK, Shrivastava S: The incidence and patterns of pulmonary artery involvement in Takayasu's arteritis. Clinical Radiology 42: 177-81, 1990.

29. Koyabu S, Isaka N, Yada T, Konishi T, Nakano T: Severe respiratory failure caused by recurrent pulmonary hemorrhage in Takayasu's arteritis. Chest 104: 1905-6, 1993.

30. Hara M, Sobue R, Ohba $S$ et al: Diffuse pulmonary lesions in early phase of Takayasu's arteritis predominantly involving pulmonary artery. J Comput Assist Tomogr 22: 801-3, 1998.

31. Liang P, Tan-Ong M, Hoffman GS: Takayasu's arteritis: vascular interventions and outcomes. J Rheumatol 31: 102-6, 2004.

32. Hunder GG, Arend WP, Bloch DA et al: The American College of Rheumatology 1990 criteria for the classification of Takayasu's arteritis. Arthritis Rheum 33: 1129-34, 1990.

33. Jennette JC, Falk RJ, Andrassy Ket al: Nomenclature of systemic vasculitides: proposal of an International Consensus Conference. Arthritis Rheum 37: 187-92, 1994.

34. Sato EI, Lima DSN, Espírito Santo B, Hatta F: Takayasu's arteritis. Treatment and prognosis in an University Center in Brazil. Int J Cardiol 75: S163-S166, 2000.

35. Sato EI, Hatta FS, Levy-Neto M, Fernandes S: Demographic, clinical and angiographic data of patients with Takayasu's arteritis in Brazil. Int J Cardiol 66(Suppl 1): S67: -S70, 1998. 